\title{
Obesity in 21-hydroxylase deficient patients
}

\author{
Rodica E Cornean, Peter C Hindmarsh, Charles G D Brook
}

\begin{abstract}
Objectives-To evaluate the natural history and timing of adiposity rebound (nadir of body mass index (BMI)) in children with congenital adrenal hyperplasia 21-hydroxylase deficiency (CYP21).

Study design-A retrospective mixed longitudinal study.

Methods-Height and changes in body composition (BMI; weight (kg)/height ${ }^{2}$ (m)), triceps and subscapular skinfolds) were analysed in 22 (14 girls, eight boys) prepubertal patients with CYP21 for whom continuous anthropometric data were available for at least seven years. BMI and height SD scores were compared at 1,5 , and 10 years of age. Skinfold SD scores were compared at 2.5 and 5.5 years. Thirteen children (nine girls, four boys) had records available from birth which allowed the estimation of the age at adiposity "peak" and "rebound".

Results-A significant increase in BMI SD score was found at 5 and 10 years compared with those at 1 year. No significant change in height SD score was observed at these ages. Triceps and skinfold SD score were increased significantly at 5.5 compared with 2.5 years. The "rebound" in BMI SD score took place at 1.74 years (range 0.71-4.57) compared with 5.5 years (range 3.5-7.0) in the normal UK population.

Conclusions-Normally growing patients with CYP21 increased their BMI throughout childhood. Adiposity rebound took place on average three years earlier than in the general population. These findings suggest that even when well controlled in terms of their disease process, patients with CYP21 are at risk of obesity, which may have important implications for the evolution of reproductive function (polycystic ovaries), diabetes, hypertension, and cardiovascular disease in these subjects.

(Arch Dis Child 1998;78:261-263)
\end{abstract}

London Centre for Paediatric Endocrinology, Middlesex Hospital, London

R E Cornean

P C Hindmarsh

C G D Brook

Correspondence to: Professor C G D Brook, Middlesex Hospital, Mortimer Street, London WIN 8AA.

Accepted

30 September 1997 has been attributed to poor therapeutic management. long term management of these children.
In normal children, body mass index (BMI) increases rapidly to a peak in infancy, then decreases before increasing again in late childhood. ${ }^{2}$ Several studies have shown that the age at which the decrease reverses (age at "adiposity rebound") is a reliable indicator of future adult obesity. The earlier the rebound, the greater the risk of obesity, regardless of the subject's adiposity at 1 year. ${ }^{3}$ There are few data on body composition in children with CYP21 and none has included the assessment of changes in BMI during this specific time interval. The aim of this study was to evaluate the natural history and timing of "adiposity rebound" in 22 patients with CYP21, all of whom were selected on the grounds of continuous "good" control as reflected by normal growth and skeletal maturation.

\section{Methods}

The longitudinal growth records of 22 (14 girls, eight boys) children with CYP21 who attended the endocrine clinic at the Middlesex Hospital were analysed. The selection criterion was the availability of continuous anthropometric growth data for at least seven years. The mean age at the time of the analysis was 12.6 years (range 7.1-20.4 years). All had CYP21 confirmed by genetic and biochemical analysis. All but three were salt losers.

Doses of glucocorticoid (hydrocortisone 15-25 $\mathrm{mg} / \mathrm{m}^{2} /$ day) and mineralocorticoid (fludrocortisone $150 \mu \mathrm{g} / \mathrm{m}^{2} /$ day) replacement were established initially using biochemical indices and adjusted thereafter according to body surface area, growth rate, and changes in skeletal maturation. Maintaining a 50th centile height velocity for chronological age with a lack of acceleration of skeletal maturation was taken as evidence of good long term control. ${ }^{4}$

Standard auxological assessment was performed at each clinic attendance at three months for the first one to two years of life, and every six months thereafter. All anthropometric data were analysed retrospectively and reported as SD scores for chronological age. Bone age was assessed using the TannerWhitehouse mark 2 method (TW2) and the values at 5 and 10 years were achieved by extrapolation. BMI values were calculated as Quetelet index (weight $(\mathrm{kg}) /$ height $^{2}(\mathrm{~m})$ ) and expressed as SD score using the UK reference data. ${ }^{2}$

Cross sectional values of BMI SD score, height, and height velocity SD score estimated at 1,5 , and 10 years were compared by one way analysis of variance (ANOVA with Student Newman Keuls post hoc test). Triceps and subscapular skinfold SD scores were compared at 2.5 and 5.5 years. The testing of differences between the two groups was performed by 


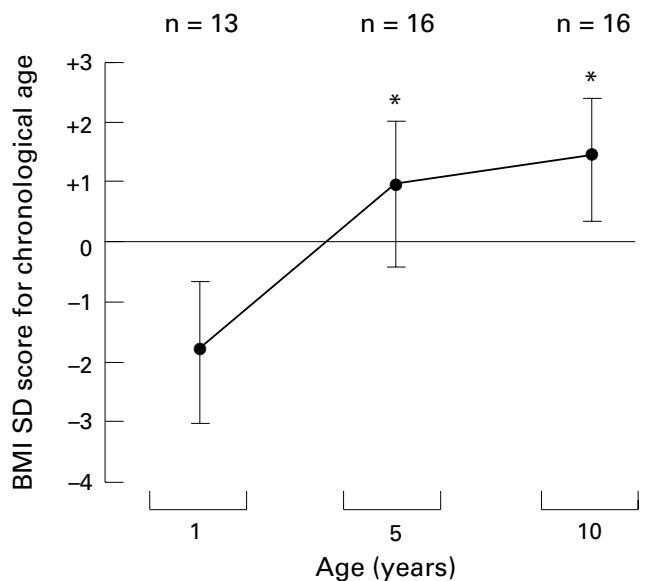

Figure 1 Changes in BMI SD scores with age in patients with 21-hydroxylase congenital adrenal hyperplasia. Data are mean $(S D)$.

means of Student's $t$ test for paired data. For 13 children (nine girls, four boys) with longitudinal records available from birth, the median BMI values for peak and trough adiposity were calculated and compared with those reported in the UK population. ${ }^{2}$

BMI SD scores were also calculated for age at the time of analysis and multiple regression was used to determine the influence of age at adiposity rebound and replacement treatment doses on BMI SD score.

\section{Results}

Figure 1 shows the BMI SD score of the study population at 1,5 , and 10 years. A significant increase in BMI SD score was found at 5 and 10 years compared with those at 1 year (one way ANOVA, $p=0.001$ ). Although the height SD score increased slightly with age, no significant change was observed between any of the above mentioned age groups for height SD score (one way ANOVA, $\mathrm{p}=0.08$ ) or height velocity SD score (one way ANOVA, $p=0.06$ ), implying normal growth for the duration of the study (fig 2). Skeletal maturation was delayed on average 0.96 "years" at 5 years (range 0.5 2.59 years) and 0.98 "years" at 10 years (range 0.56-3.02 years).

The increase in skinfold thicknesses for skinfold and triceps SD score mirrored the changes observed in BMI, with a statistically significant increase in values between 2.5 and 5.5 years. The difference between the means and 95\% confidence interval for the two parameters were: skinfold SD score 1.75 (0.556 to 2.944), Student's paired $t$ test, $\mathrm{p}=0.01$; triceps SD score 1.41 ( -0.15 to 2.852$)$; Student's paired $t$ test, $\mathrm{p}=0.05$.

For the group of children with measurements available from birth, individual longitudinal BMI curves were plotted. The median peak BMI SD score $(-0.14$ (range -3.21 to $+1.160)$ ) was attained at 6.8 months (normal age 8 months). The median BMI SD score at rebound was -1.95 (range -4.650 to +0.05 ) and this was attained at 1.74 years (range 0.71 to 4.57 ) compared with 5.5 years (range 3.5 to 7.0) in the UK population. ${ }^{2}$ The distribution of age at adiposity rebound was skewed towards the earlier age.
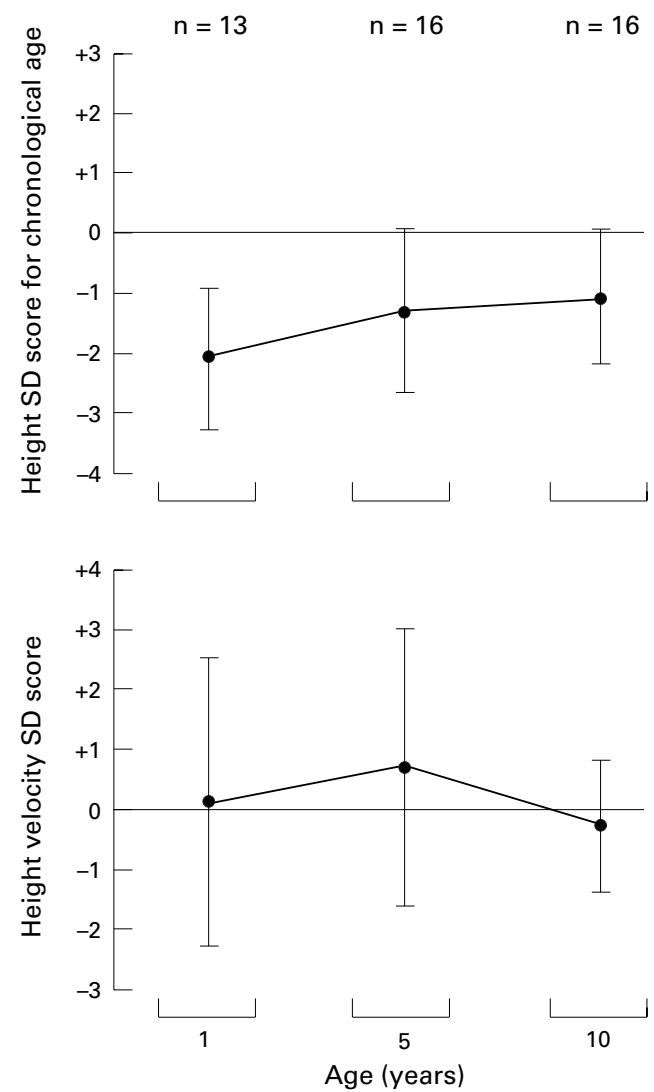

Figure 2 Changes in height (upper panel) and height velocity $S D$ scores (lower panel) with age in patients with 21-hydroxylase congenital adrenal hyperplasia. Data are mean $(S D) . p=N S$ compared with values at 1 year of age.

The mean age of the patients when the analysis was performed was 12.6 years (range 7.1 to 20.4), by which time the median BMI SD score was 1.51 (range -0.73 to 3.61 ). The mean doses and range for hydrocortisone and fludrocortisone replacement were $18.96 \mathrm{mg} /$ $\mathrm{m}^{2} /$ day (range 15.52 to 23.12 ) and $132 \mu \mathrm{g} / \mathrm{m}^{2} /$ day (range 98 to 162.0 ). These ranges and those for adiposity rebound were too narrow to explore potential interactions.

\section{Discussion}

Overtreatment with glucocorticoids, either as replacement in patients with CYP21 or in the management of inflammatory disorders in paediatrics, can result in truncal obesity, osteopenia, and Cushing's syndrome. Furthermore, hyperandrogenism in children with 21hydroxylase deficiency who have escaped suppression as a result of undertreatment or erratic compliance with the prescribed glucocorticoid and mineralocorticoid treatment may account for the increase in BMI and loss of growth potential. ${ }^{6}$ However, little is known about the effect of glucocorticoids in modulating body composition in congenital adrenal hyperplasia, when optimum control (in terms of linear growth) has been achieved with conventional replacement regimens.

Our data show that the treatment of children with CYP21 with glucocorticoids (15-25 $\mathrm{mg} / \mathrm{m}^{2} /$ day $)$ and fludrocortisone $\left(0.15 \mathrm{mg} / \mathrm{m}^{2} /\right.$ day) to promote normal linear growth resulted 
in significantly higher values for BMI throughout childhood. This index is clearly affected by height, weight, and body composition. The increase in BMI in our patients was due to a change in body weight because the height SD score remained constant, and the weight gain was attributable to fat mass as reflected by increases in skinfold thickness. Adiposity "rebound" took place in these patients with congenital adrenal hyperplasia about three years earlier than is normally seen in the UK population.

The increases in BMI at ages 5 and 10 years in patients with CYP21 are consistent with the observation that early "rebound" of BMI is associated with obesity in late childhood. The changes in body composition in our group of patients with CYP21 were in sharp contrast with the optimum height achieved for the duration of the study. Although conventional replacement treatment in these patients may have been optimum with regard to longitudinal growth, body composition was adversely affected.

The current BMI SD scores were greater than zero in all but one subject. A variance in the dose of hydrocortisone at this age was too small to determine the impact on fatness. The distribution of age at rebound was positively skewed with a narrow variance, so that a causeeffect relation between BMI SD score and dose of hydrocortisone or age at adiposity rebound, or both, was not evident.

Our findings suggest that patients with CYP21 are at risk of later obesity and this may have important implications for long term health problems in these patients; obesity is associated with polycystic ovaries, diabetes, hypertension, and cardiovascular disease.

The increase in weight throughout childhood may arise because of the fact that glucocorticoid replacement treatment does not mimic the normal circadian pattern of cortisol secretion $^{7}$ or may be due to the suppression of growth hormone, which is known to be associated with an increase in body fat, or a combination of both mechanisms. Longer acting glucocorticoids, which achieve more persistent glucocorticoid exposure-for example, dexamethasone-will only exacerbate this effect. These observations suggest that careful consideration needs to be given to steroid replacement in these patients, with both weight and height gain balanced carefully in the overall replacement equation. Attention should be paid to adiposity rebound in individual patients.

We conclude that in patients with CYP21 who are growing normally, an increase in BMI throughout childhood occurred as a result of an increase in body fat. The reasons for this require further research but, as they are at odds with our experience in patients with Addison's disease, the present findings support the argument that patients with CYP21 might do better without their adrenal glands. ${ }^{8}$

1 Knorr D, Hinrichsen de Lienan SGC. Persistent obesity and short final height after corticoid overtreatment for congenital adrenal hyperplasia in infancy. Acta Paediatr fpn tal adrenal hyp

2 Cole TJ, Freeman JV, Preece MA. Body mass index reference curves for UK, 1990. Arch Dis Child 1995;73:259.

3 Rolland-Cachera MF, Deheeger M, Bellisle F, et al. Adiposty rebound in children: a simple indicator for predicting obesity. Am f Clin Nutr 1984;39:129-35.

4 Appan S, Hindmarsh PC, Brook CGD. Monitoring treatment in congenital adrenal hyperplasia. Arch Dis Child 1989;64:1235-9.

5 Gasparini N, Di Maio S, Salerno M, et al. Growth pattern during the first 36 months of life in congenital adrenal hyperplasia (21-hydroxylase deficiency). Horm Res 1997; 47:17-22.

$6 \mathrm{Yu}$ ACM, Grant DB. Adult height in women with Yu ACM, Grant DB. Adult height in women with hydroxylase type): relation to body mass index in earlier childhood. Acta Paediatr 1995;84:899-903.

7 De Vile CJ, Stanhope R. Hydrocortisone replacement therapy in children and adolescents with hypopituitarism Clin Endocrinol (Oxf) 1997;47:37-41.

8 Van Wyk JJ, Gunther DF, Ritzen EM, et al. The use of adrenalectomy as a treatment for congenital adrenal hyperplasia. F Clin Endocrinol Metab 1996;81:3180-90. 